EPJ Web of Conferences 38, 09005 (2012)

DOI: $10.1051 /$ epjconf/20123809005

(C) Owned by the authors, published by EDP Sciences, 2012

\title{
Role of neutron transfer and deformation effect in capture process at sub-barrier energies
}

\author{
V. V. Sargsyan ${ }^{1, a}$, G. G. Adamian ${ }^{1}$, N. V. Antonenko ${ }^{1}$, W. Scheid ${ }^{2}$, and H. Q. Zhang ${ }^{3}$ \\ 1 Bogoliubov Laboratory of Theoretical Physics, JINR, 141980 Dubna, Russia \\ 2 Institut für Theoretische Physik der Justus-Liebig-Universität, D-35392 Giessen, Germany \\ 3 China Institute of Atomic Energy, Post Office Box 275, Beijing 102413, China
}

\begin{abstract}
The roles of nuclear deformation and neutron transfer in sub-barrier capture process are studied within the quantum diffusion approach. The change of the deformations of colliding nuclei with neutron exchange can crucially influence the sub-barrier fusion. The sub-barrier capture reactions following the neutron pair transfer are used for the indirect study of neutron-neutron correlation in the surface region of nucleus. The strong surface enhancement of the neutron pairing in nuclei ${ }^{48} \mathrm{Ca},{ }^{64} \mathrm{Ni}$, and ${ }^{116,124,132} \mathrm{Sn}$ is demonstrated. Comparing the capture cross sections calculated without the breakup effect and experimental complete fusion cross sections, the breakup was analyzed in reactions with weakly bound projectiles ${ }^{6,7,9} \mathrm{Li}$ and ${ }^{9} \mathrm{Be}$. A trend of a systematic behavior for the complete fusion suppression as a function of the target charge and bombarding energy is not achieved.
\end{abstract}

\section{Introduction}

The nuclear deformation and neutron-transfer process have been identified as playing a major role in the magnitude of the sub-barrier capture and fusion cross sections [1,2]. There are a several experimental evidences which confirm the importance of nuclear deformation on the capture and fusion. The influence of nuclear deformation is straightforward. If the target nucleus is prolate in the ground state, the Coulomb field on its tips is lower than on its sides, that then increases the capture or fusion probability at energies below the barrier corresponding to the spherical nuclei. The role of neutron transfer reactions is less clear. A correlation between the overall transfer strength and fusion enhancement was firstly noticed in Ref. [3]. The importance of neutron transfer with positive $Q$-values on nuclear fusion (capture) originates from the fact that neutrons are insensitive to the Coulomb barrier and therefore they can start being transferred at larger separations before the projectile is captured by target-nucleus [4]. Therefore, it is generally thought that the sub-barrier fusion cross section will increase [5-7] because of the neutron transfer. As suggested in Ref. [8], the enhancements in fusion yields may be due to the transfer of a neutron pair with a positive $Q$-value. However, as shown recently in Ref. [9], the two-neutron transfer channel with large positive $Q$-value weakly influences the fusion (capture) cross section in the ${ }^{60} \mathrm{Ni}+{ }^{100}$ Mo reaction at sub-barrier energies. So, from the present data an unambiguous signature of the role of neutron transfer channel could not be inferred. Another open question is the nucleon pairing correlations in the stable nuclei. The corresponding pair transfer modes are usually described in terms of pairing vibrations or pairing rotations $[10,11]$, which are one of collective vibrational modes associated with the pair correlation. It has been established that the two-neutron transfer amplitude is

\footnotetext{
a e-mail: sargsyan@theor. jinr.ru
}

influenced by collective modes caused by the Cooper-pair superfluidity [12]. The experimental signatures of a spatial two-neutron correlation or the di-neutron correlation between two weakly bound neutrons forming the halo in ${ }^{6,8} \mathrm{He}$ and ${ }^{11} \mathrm{Li}$ have been reported in Refs. [13-16].

In the present paper the quantum diffusion approach[17, $18]$ is applied to study the fusion hindrance and the roles of nuclear deformation and neutron transfer in sub-barrier capture process. Also, we will consider capture reactions with the negative $\left(Q_{1 n}<0\right)$ one-neutron transfer and the positive $\left(Q_{2 n}>0\right)$ two-neutron transfer (before the crossing of the Coulomb barrier), where the one-step neutron pair transfer is expected to prevail. The study of this process is one of the key point in the understanding of pairing correlations in nuclei. The distinction between two-step sequential and one-step cluster transfer is a great challenge, not only in nuclear physics but also in electron transfer between ions or atomic cluster collisions [12]. Note that the capture (fusion) reaction following the neutron pair transfer is the indirect way of the study on pairing effects, and completely different from the two-neutron or multinucleon transfer reaction.

\section{Model}

In the quantum diffusion approach the collisions of nuclei are treated in terms of a single collective variable: the relative distance between the colliding nuclei. The nuclear deformation effects are taken into consideration through the dependence of the nucleus-nucleus potential on the deformations and orientations of colliding nuclei. Our approach takes into consideration the fluctuation and dissipation effects in collisions of heavy ions which model the coupling with various channels. We have to mention that many quantum-mechanical and non-Markovian effects accompanying the passage through the potential barrier are taken into consideration in our formalism [17,19,20]. The 
details of used formalism are presented in our previous articles $[17,18]$. All parameters of the model are set as in Ref. [17]. All calculated results are obtained with the same set of parameters and are rather insensitive to the reasonable variation of them $[17,18]$. The heights of the calculated Coulomb barriers $V_{b}=V\left(R_{b}\right)\left(R_{b}\right.$ is the position of the Coulomb barrier) are adjusted to the experimental data for the fusion or capture cross sections. To calculate the nucleus-nucleus interaction potential $V(R)$, we use the procedure presented in Refs. [17,18]. For the nuclear part of the nucleus-nucleus potential, the double-folding formalism with the Skyrme-type density-dependent effective nucleon-nucleon interaction is used.

\section{Effect of quadrupole deformation}

To separate the effects of deformation and neutron transfer, firstly we consider the reactions with deformed nuclei in which $Q$-value for the neutron transfer are small, i.e. the neutron transfers can be disregarded. In figures 1 and 2 , the calculated capture cross sections for the reactions ${ }^{16} \mathrm{O},{ }^{48} \mathrm{Ca},{ }^{40} \mathrm{Ar}+{ }^{154} \mathrm{Sm}$, and ${ }^{74} \mathrm{Ge}+{ }^{74} \mathrm{Ge}$ are in a good agreement with the available experimental data [21-24] showing that the quadrupole deformations of the interacting nuclei are the main reasons for the enhancement of the capture cross section at sub-barrier energies. The quadrupole deformation parameters $\beta_{2}$ are taken from Ref. [25] for the deformed even-even nuclei. In Ref. [25] the quadropole deformation parameters $\beta_{2}$ for the first excited $2^{+}$states of nuclei are given. For the nuclei deformed in the ground state, the $\beta_{2}$ in $2^{+}$state is similar to the $\beta_{2}$ in the ground state and we use $\beta_{2}$ from Ref. [25] in the calculations. For double magic nuclei, in the ground state we take $\beta_{2}=0$. In Ref. [26] the experimentally observed enhancement of sub-barrier fusion for the reactions ${ }^{16} \mathrm{O},{ }^{48} \mathrm{Ca}+{ }^{154} \mathrm{Sm}$, and ${ }^{74} \mathrm{Ge}+{ }^{74} \mathrm{Ge}$ was explained by the nucleon transfer and neck formation effects. However, in the present article we demonstrate that a good agreement with the experimental data at sub-barrier energies could be reached taking only the quadrupole deformations of interacting nuclei into consideration. We should mention, that for the sub-barrier energies the results of calculations are very sensitive to the quadrupole deformation parameters $\beta_{2}$ of the interacting nuclei. Since there are uncertainties in the definition of the values of $\beta_{2}$ in the light- and the medium-mass nuclei, one can extract the quadrupole deformation parameters of these nuclei from the comparison of the calculated capture cross sections with the experimental data.

\section{Effect of neutron transfer}

Several experiments were performed to understand the effect of neutron transfer in the fusion (capture) reactions. The choice of the projectile-target combination is crucial, and for the systems studied one can make unambiguous statements regarding the neutron transfer process with a positive $Q$-value when the interacting nuclei are double magic or semi-magic spherical nuclei. In this case one can disregard the strong nuclear deformation effects. The good examples are the reactions with the spherical nuclei: ${ }^{40} \mathrm{Ca}$ $+{ }^{208} \mathrm{~Pb}\left(Q_{2 n}=5.7 \mathrm{MeV}\right)$ and ${ }^{40} \mathrm{Ca}+{ }^{96} \mathrm{Zr}\left(Q_{2 n}=5.5 \mathrm{MeV}\right)$.
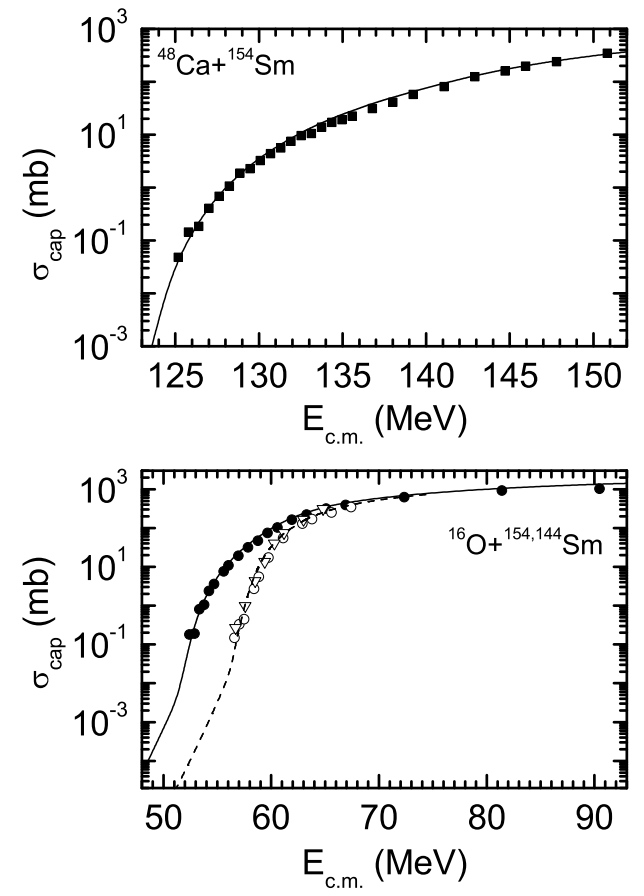

Fig. 1. The calculated capture cross sections versus $E_{\text {c.m. }}$ for the indicated reactions ${ }^{16} \mathrm{O},{ }^{48} \mathrm{Ca}+{ }^{154} \mathrm{Sm}$ (solid lines), and ${ }^{16} \mathrm{O}$ $+{ }^{144} \mathrm{Sm}$ (dashed line). The experimental data (symbols) are from Refs. [21,22,27]. The following quadrupole deformation parameters are used: $\beta_{2}\left({ }^{154} \mathrm{Sm}\right)=0.341[25], \beta_{2}\left({ }^{144} \mathrm{Sm}\right)=0.05$, and $\beta_{2}\left({ }^{16} \mathrm{O}\right)=\beta_{2}\left({ }^{48} \mathrm{Ca}\right)=0$.
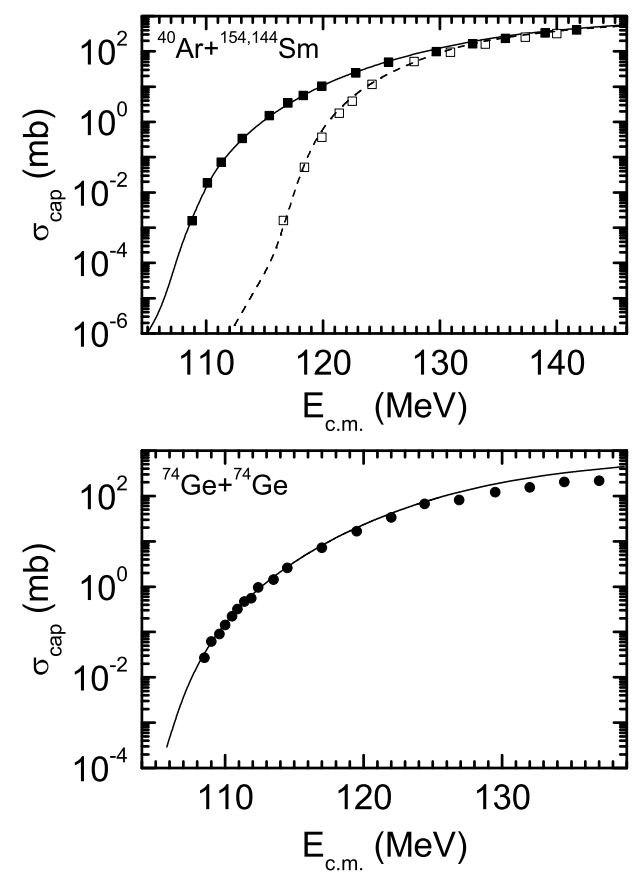

Fig. 2. The same as figure 1 , for the indicated reactions ${ }^{74} \mathrm{Ge}+{ }^{74} \mathrm{Ge},{ }^{40} \mathrm{Ar}+{ }^{154} \mathrm{Sm}$ (solid lines), and ${ }^{40} \mathrm{Ar}+{ }^{144} \mathrm{Sm}$ (dashed line). The experimental data (symbols) are from Ref. [23,24]. The following quadrupole deformation parameters are used: $\beta_{2}\left({ }^{40} \mathrm{Ar}\right)=0.25$ [25], $\beta_{2}\left({ }^{74} \mathrm{Ge}\right)=0.2825$ [25], $\beta_{2}\left({ }^{154} \mathrm{Sm}\right)=0.341[25]$, and $\beta_{2}\left({ }^{144} \mathrm{Sm}\right)=0.05$. 

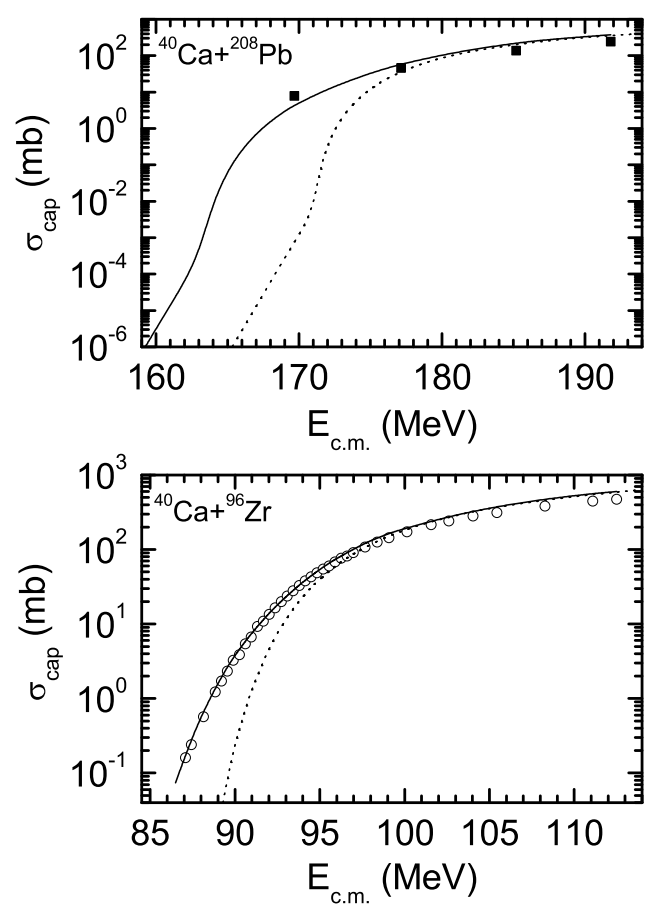

Fig. 3. The same as figure 1 , for the indicated reactions ${ }^{40} \mathrm{Ca}$ $+{ }^{208} \mathrm{~Pb},{ }^{96} \mathrm{Zr}$ (solid lines). The calculated capture cross sections without taking into consideration the neutron transfer process are shown by dotted lines. The experimental data (symbols) are from Refs. [28,29]. The following quadrupole deformation parameters are used: $\beta_{2}\left({ }^{42} \mathrm{Ca}\right)=0.247$ [25], $\beta_{2}\left({ }^{94} \mathrm{Zr}\right)=0.09$ [25], $\beta_{2}\left({ }^{96} \mathrm{Zr}\right)=0.08$, and $\beta_{2}\left({ }^{40} \mathrm{Ca}\right)=\beta_{2}\left({ }^{206,208} \mathrm{~Pb}\right)=0$.

Since the transfer of protons is shielded by the Coulomb barrier, it occurs when two nuclei almost touch each other [30], i.e. after a capture. Thus, the proton transfer can be disregarded in the calculations of capture cross sections. Following the hypothesis of Ref. [8], we assume that the sub-barrier capture mainly depends on the two-neutron transfer with the positive and relatively large $Q$-value. Our assumption is that, before the projectile is captured by targetnucleus (before the crossing of the Coulomb barrier) which is the slow process, the two-neutron transfer occurs at larger separations that can lead to the population of the first $2^{+}$ state in the recipient nucleus [31].

Since after two-neutron transfer the mass numbers, the deformation parameters of interacting nuclei, and, respectively, the height and shape of the Coulomb barrier are changed, one can expect the enhancement or suppression of the capture. For example, after the neutron transfer in the reaction ${ }^{40} \mathrm{Ca}\left(\beta_{2}=0\right)+{ }^{208} \mathrm{~Pb}\left(\beta_{2}=0\right) \rightarrow{ }^{42} \mathrm{Ca}\left(\beta_{2}=0.247\right)$ $+{ }^{206} \mathrm{~Pb}\left(\beta_{2}=0\right)\left({ }^{40} \mathrm{Ca}\left(\beta_{2}=0\right)+{ }^{96} \mathrm{Zr}\left(\beta_{2}=0.08\right) \rightarrow{ }^{42} \mathrm{Ca}\right.$ $\left.\left(\beta_{2}=0.247\right)+{ }^{94} \mathrm{Zr}\left(\beta_{2}=0.09\right)\right)$ the deformation of the nuclei increases and the mass asymmetry of the system decreases and thus the value of the Coulomb barrier decreases and the capture cross section becomes larger (figure 3). One can see a good agreement between the calculated results and the experimental data. For some reactions at energies above the Coulomb barrier, the small deviation between the calculated results and experimental data
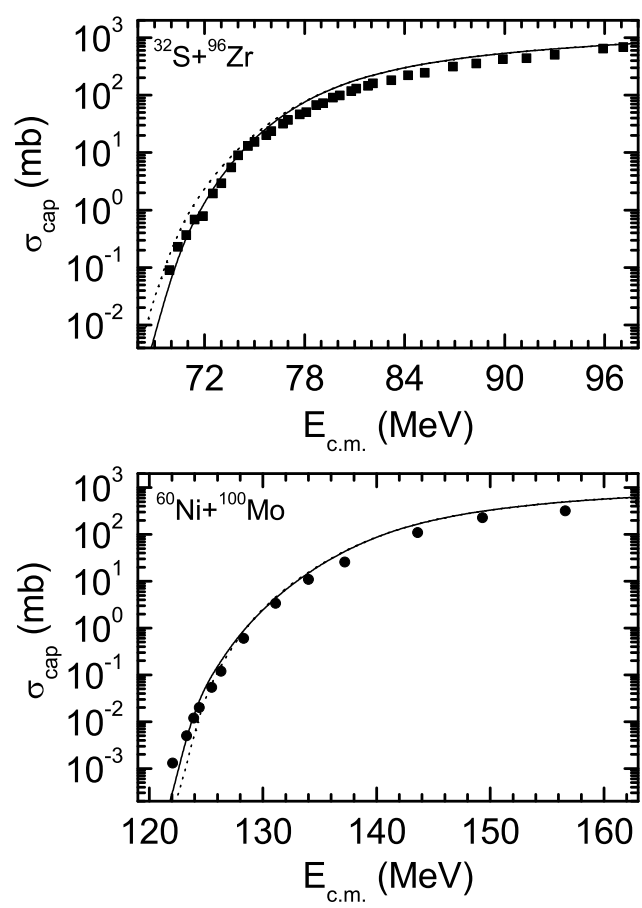

Fig. 4. The same as figure 1 , for the indicated reactions ${ }^{32} \mathrm{~S}+$ ${ }^{96} \mathrm{Zr}$, and ${ }^{60} \mathrm{Ni}+{ }^{100} \mathrm{Mo}$ (solid lines). The experimental data (symbols) are from Refs. [32,9]. The following quadrupole deformation parameters are used: $\beta_{2}\left({ }^{32} \mathrm{~S}\right)=0.312[25], \beta_{2}\left({ }^{34} \mathrm{~S}\right)=0.252$ [25], $\beta_{2}\left({ }^{96} \mathrm{Zr}\right)=0.08$, and $\beta_{2}\left({ }^{36} \mathrm{~S}\right)=\beta_{2}\left({ }^{90} \mathrm{Zr}\right)=0$.

probably arises from the fact that the fusion-fission channel was not taken into consideration in the experimental capture cross sections. So, our results show that the observed capture enhancement at sub-barrier energies for the reactions mentioned above is related to the two-neutron transfer channel.

Note that strong population of the yrast states, and in particular of the first $2^{+}$state of even $\mathrm{Ca}$ isotopes via the neutron pick-up channels in the ${ }^{40} \mathrm{Ca}+{ }^{96} \mathrm{Zr}$ reaction is experimentally found in Ref. [31]. In the calculations, for such excited recipient nuclei we use the experimental deformation parameters $\beta_{2}$ related to the first $2^{+}$states from the table of Ref. [25]. We assume that after two neutron transfer the residues of donor nuclei remain in the ground state with corresponding quadrupole deformation.

One can find the reactions with large positive two-neutron transfer $Q$-values where the transfer weakly influences or even suppresses the capture process. This happens if after transfer the deformations of nuclei almost do not change or even decrease. For instance, in the reactions

${ }^{32} \mathrm{~S}\left(\beta_{2}=0.312\right)+{ }^{96} \mathrm{Zr}\left(\beta_{2}=0.08\right) \rightarrow{ }^{34} \mathrm{~S}\left(\beta_{2}=0.252\right)+$ ${ }^{94} \mathrm{Zr}\left(\beta_{2}=0.09\right)$ and ${ }^{60} \mathrm{Ni}\left(0.05<\beta_{2} \lesssim 0.1\right)+{ }^{100} \mathrm{Mo}\left(\beta_{2}=\right.$ $0.231) \rightarrow{ }^{62} \mathrm{Ni}\left(\beta_{2}=0.198\right)+{ }^{98} \mathrm{Mo}\left(\beta_{2}=0.168\right)$ one can expect weak dependence of the capture cross section on the neutron transfer (figure 4). There is the experimental indication of such effect for the ${ }^{60} \mathrm{Ni}+{ }^{100}$ Mo reaction [9]. 

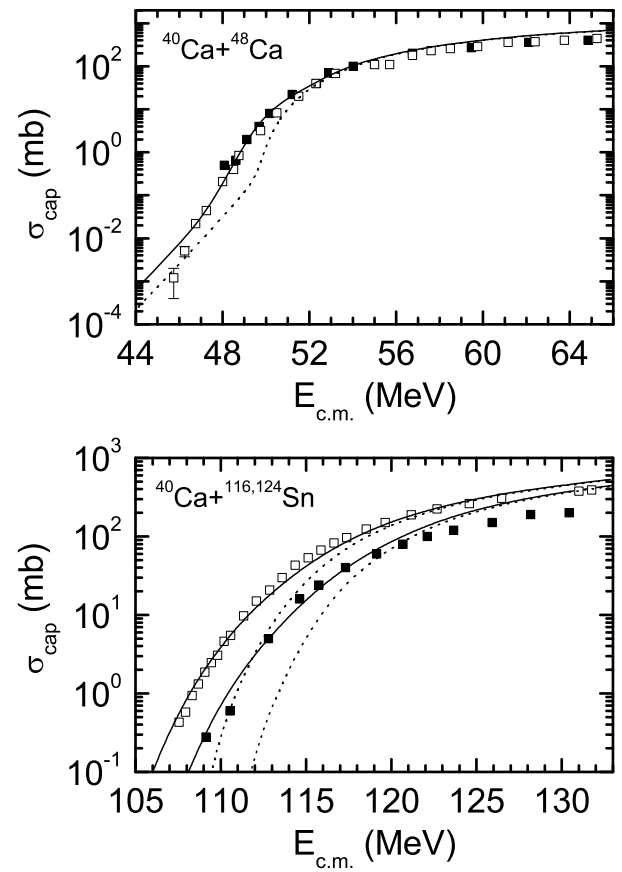

Fig. 5. The same as figure 1, for the indicated reactions ${ }^{40} \mathrm{Ca}+{ }^{48} \mathrm{Ca},{ }^{116,124} \mathrm{Sn}$ (solid lines). The calculated capture cross sections without taking into consideration the neutron transfer process are shown by dotted lines. The experimental data (symbols) are from Refs. [33,34]. The following quadrupole deformation parameters are used: $\beta_{2}\left({ }^{42} \mathrm{Ca}\right)=0.247 \quad$ [25], $\beta_{2}\left({ }^{116} \mathrm{Sn}\right)=0.112 \quad[25], \quad \beta_{2}\left({ }^{114} \mathrm{Sn}\right)=0.121, \quad \beta_{2}\left({ }^{124} \mathrm{Sn}\right)=0.095$, $\beta_{2}\left({ }^{122} \mathrm{Sn}\right)=0.104$, and $\beta_{2}\left({ }^{40} \mathrm{Ca}\right)=\beta_{2}\left({ }^{46,48} \mathrm{Ca}\right)=0$.

\section{Neutron pair transfer in sub-barrier capture process}

There exists several studies demonstrating enhancement of the pair correlation in the nuclear surface and exterior regions of the neutron-rich nuclei [35-38]. The effect of correlations between nucleons on the nuclear breakup mechanism has been studied both experimentally and theoretically $[39,40]$. There exists also several studies demonstrating enhancement of the pair correlation in the nuclear surface and exterior regions of the neutron-rich nuclei [3538].

By describing the capture (fusion) reactions at sub-barrier energies within the quantum diffusion approach, we want to demonstrate indirectly the strong surface enhancement of the neutron pairing in the stable and unstable nuclei. We will consider capture reactions with the negative $\left(Q_{1 n}<0\right)$ one-neutron transfer and the positive $\left(Q_{2 n}>0\right)$ two-neutron transfer (before the crossing of the Coulomb barrier), where the one-step neutron pair transfer is expected to prevail. The study of this process is one of the key point in the understanding of pairing correlations in nuclei. The distinction between two-step sequential and one-step cluster transfer is a great challenge, not only in nuclear physics but also in electron transfer between ions or atomic cluster collisions [12]. Note that the capture (fusion) reaction following the neutron pair transfer is an indirect way of the study on pairing effects, and completely
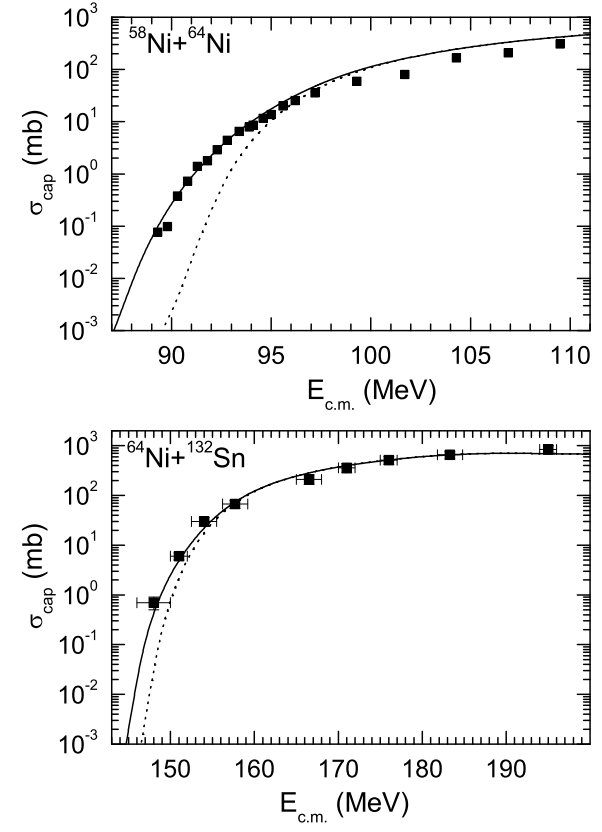

Fig. 6. The same as figure 1, for the indicated reactions ${ }^{58} \mathrm{Ni}+{ }^{64} \mathrm{Ni}$ and ${ }^{64} \mathrm{Ni}+{ }^{132} \mathrm{Sn}$ (solid lines). The calculated capture cross sections without taking into consideration the neutron transfer process are shown by dotted lines. The experimental data (symbols) are from Refs. [41, 42]. The following quadrupole deformation parameters are used: $\beta_{2}\left({ }^{58} \mathrm{Ni}\right)=0.05$ [25], $\beta_{2}\left({ }^{64} \mathrm{Ni}\right)=0.087$ [25], $\beta_{2}\left({ }^{60} \mathrm{Ni}\right)=0.207$, $\beta_{2}\left({ }^{62} \mathrm{Ni}\right)=0.087, \beta_{2}\left({ }^{66} \mathrm{Ni}\right)=0.158$, and $\beta_{2}\left({ }^{132} \mathrm{Sn}\right)=\beta_{2}\left({ }^{130} \mathrm{Sn}\right)=0$.

different from the two-neutron or multinucleon transfer reaction. We will show that the information obtained from the sub-barrier capture (fusion) reactions is complementary to that obtained from the two-neutron transfer reactions such as $(p, t)$ or $(t, p)$.

The choice of the projectile-target combination is crucial in the understanding of pair tunneling phenomena in the capture process. In the capture reactions with $Q_{1 n}<0$ and $Q_{2 n}>0$, the two-step sequential transfer is almost closed before capture. So, by properly choosing the reaction combination, one can reduce the role successive transfer plays in the process. For the systems studied one can make unambiguous statements regarding the neutron transfer process with a positive $Q_{2 n}$ value when the interacting nuclei are double magic or semimagic nuclei. In this case one can disregard the strong nuclear deformation effects before the neutron transfer. In figures 5 and 6 the calculated capture cross sections for the reactions ${ }^{40} \mathrm{Ca}+{ }^{48} \mathrm{Ca}$ $\left(Q_{1 n}=-1.6 \mathrm{MeV}, Q_{2 n}=2.6 \mathrm{MeV}\right),{ }^{40} \mathrm{Ca}+{ }^{116} \mathrm{Sn}\left(Q_{1 n}=\right.$ $\left.-1.2 \mathrm{MeV}, Q_{2 n}=2.8 \mathrm{MeV}\right),{ }^{40} \mathrm{Ca}+{ }^{124} \mathrm{Sn}\left(Q_{1 n}=-0.1\right.$ $\left.\mathrm{MeV}, Q_{2 n}=5.4 \mathrm{MeV}\right),{ }^{58} \mathrm{Ni}+{ }^{64} \mathrm{Ni}\left(Q_{1 n}=-0.66 \mathrm{MeV}\right.$, $\left.Q_{2 n}=3.9 \mathrm{MeV}\right)$, and ${ }^{64} \mathrm{Ni}+{ }^{132} \mathrm{Sn}\left(Q_{1 n}=-1.21 \mathrm{MeV}\right.$, $Q_{2 n}=2.5 \mathrm{MeV}$ ) are in a good agreement with the available experimental data $[33,34,41,42]$. In all reactions $1 n$ neutron transfer process are closed $\left(Q_{1 n}<0\right)$ and $Q_{2 n^{-}}$ values for the $2 n$-transfer processes are positive. Thus, the $2 n$-neutron transfer has to be taken into account for good description of the experimental data. The influence of the 
$2 n$-neutron transfer on the capture cross section occurs due to the change of the isotopic composition and the deformations of the reaction partners. The $2 n$-transfer indirectly influence the quadrupole deformation of the nuclei. When after the neutron transfer (just before the crossing of the Coulomb barrier) in the reactions ${ }^{40} \mathrm{Ca}\left(\beta_{2}=0\right)+{ }^{48} \mathrm{Ca}\left(\beta_{2}=\right.$ $0) \rightarrow{ }^{42} \mathrm{Ca}\left(\beta_{2}=0.247\right)+{ }^{46} \mathrm{Ca}\left(\beta_{2}=0\right),{ }^{40} \mathrm{Ca}\left(\beta_{2}=0\right)$ $+{ }^{116} \mathrm{Sn}\left(\beta_{2}=0.112\right) \rightarrow{ }^{42} \mathrm{Ca}\left(\beta_{2}=0.247\right)+{ }^{114} \mathrm{Sn}\left(\beta_{2}=\right.$ $0.121),{ }^{40} \mathrm{Ca}\left(\beta_{2}=0\right)+{ }^{124} \mathrm{Sn}\left(\beta_{2}=0.095\right) \rightarrow{ }^{42} \mathrm{Ca}\left(\beta_{2}=\right.$ $0.247)+{ }^{122} \mathrm{Sn}\left(\beta_{2}=0.104\right),{ }^{58} \mathrm{Ni}\left(\beta_{2}=0.05\right)+{ }^{64} \mathrm{Ni}\left(\beta_{2}=\right.$ $0.087) \rightarrow{ }^{60} \mathrm{Ni}\left(\beta_{2}=0.207\right)+{ }^{62} \mathrm{Ni}\left(\beta_{2}=0.087\right)$, and ${ }^{64} \mathrm{Ni}\left(\beta_{2}=\right.$ $0.087)+{ }^{132} \mathrm{Sn}\left(\beta_{2}=0\right) \rightarrow{ }^{66} \mathrm{Ni}\left(\beta_{2}=0.158\right)+{ }^{130} \mathrm{Sn}\left(\beta_{2}=\right.$ $0)$ the deformations of nuclei increase, the values of the corresponding Coulomb barriers decrease. As a result, the neutron transfer enhances the capture process in these reactions at the sub-barrier energies. The enhancement becomes stronger with decreasing bombarding energy (figures 5 and 6).

Since $Q_{1 n}<0$ in these reactions, the enhancement arises not from the coherent successive transfer of two single nucleons, but from the direct transfer of one spatially correlated pair (the simultaneous transfer of two nucleons). Neutron pair transfer indicates that stable nucleus at low densities around nuclear surface may exhibit features of the strong coupling pairing, characterized by the small size of the Cooper pair comparable with average internucleon distance. This may suggest a possible enhancement of the pair correlation in the surface of nuclei ${ }^{48} \mathrm{Ca},{ }^{64} \mathrm{Ni}$, and ${ }^{116,124,132} \mathrm{Sn}$. Our results show that the capture (fusion) cross section of the reactions under consideration can be described by assuming the preformed dineutron-like clusters in the ground state of these nuclei. Note that the spatial two-neutron correlation and the strong surface enhancement of the neutron pairing in the cases of a slab and a semi-infinite nuclear matter are well known and it is well established that nuclear superfluidity is mainly a surface effect [43].

\section{Reactions with weakly bound projectiles at energies around Coulomb barrier}

In recent years, many efforts have been made to understand the effect of breakup of weakly bound nuclei during the fusion reaction in very asymmetric reactions where the capture cross section is equal to the complete fusion cross section [2,44-50]. The light radioactive nuclei, especially halo nuclei, such as ${ }^{6} \mathrm{He},{ }^{8} \mathrm{~B},{ }^{11} \mathrm{Be}$, and the stable nuclei ${ }^{6} \mathrm{Li}$ and ${ }^{9} \mathrm{Be}$ are weakly bounded, hence there is a chance of the breakup in the colliding process. By performing a comparison of fusion data with theoretical predictions which do not take into account the dynamic breakup plus transfer channel effects, it has been shown [46-48, 50], that for energies from about $1.1 V_{b}$ to $1.5 V_{b}$ ( $V_{b}$ is the height of the Coulomb barrier) complete fusion in the reactions ${ }^{6,7} \mathrm{Li}+{ }^{208} \mathrm{~Pb},{ }^{209} \mathrm{Bi}$ and ${ }^{9} \mathrm{Be}+{ }^{89} \mathrm{Y},{ }^{124} \mathrm{Sn},{ }^{208} \mathrm{~Pb},{ }^{209} \mathrm{Bi}$ is suppressed by about $30 \%$. However, the ${ }^{9} \mathrm{Be}+{ }^{144} \mathrm{Sm}$ data is out of the systematics, showing a much smaller suppression of about $15 \%$. The total fusion (incomplete fusion + sequential complete fusion + complete fusion) cross section for the same projectiles on targets of any mass, including ${ }^{9} \mathrm{Be}+{ }^{27} \mathrm{Al},{ }^{64} \mathrm{Zn}$, does not seem to be affected by the dynamic breakup and transfer effects $[48,50]$. As
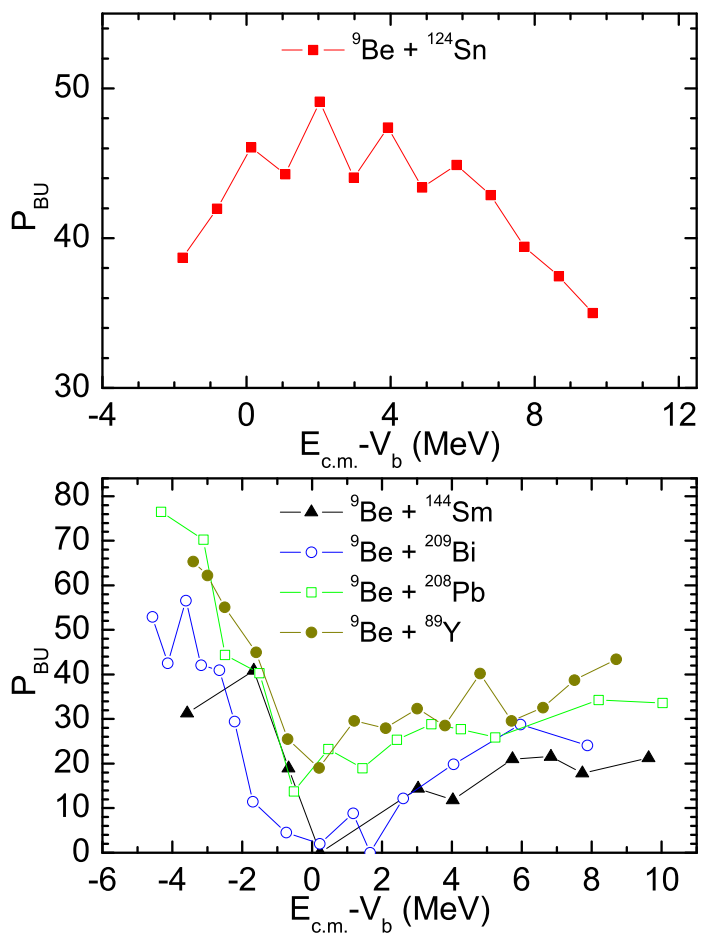

Fig. 7. The dependence of the extracted breakup probability $P_{B U}$ vs $E_{c . m .}-V_{b}$ for the indicated reactions with ${ }^{9} \mathrm{Be}$-projectiles in $\%$.

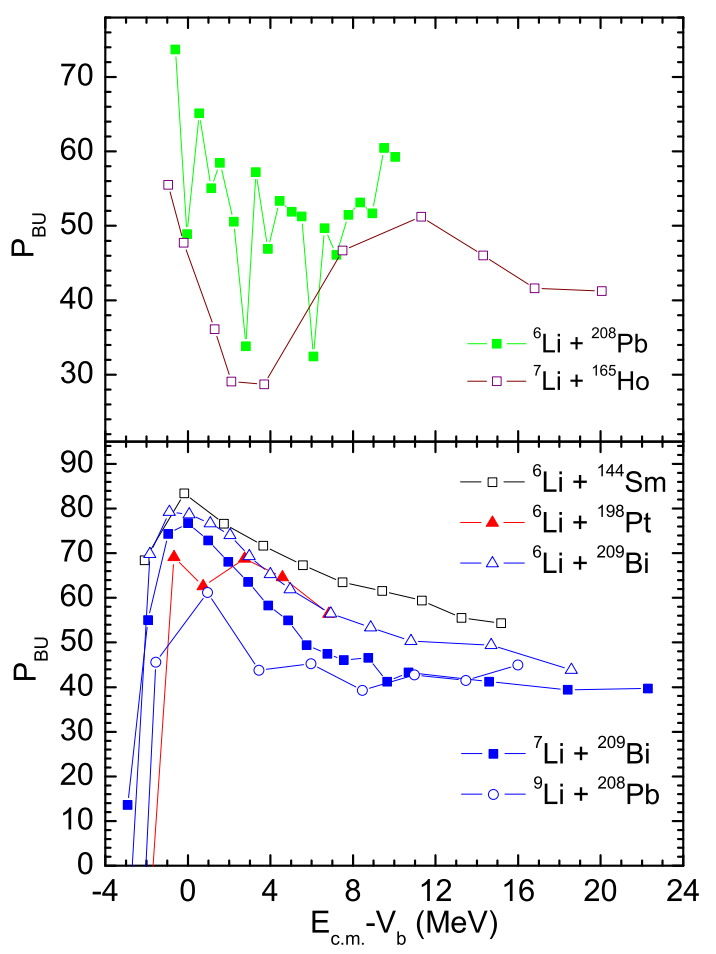

Fig. 8. The same as in figure 7 , but for the indicated reactions with ${ }^{6,7,9} \mathrm{Li}$-projectiles.

the charge of the target decreases, one expects that the Coulomb breakup becomes weaker, and consequently the complete fusion suppression and incomplete fusion probability decrease. The lack of a clear systematic behavior of the complete fusion suppression as a function of the target charge was explained in Ref. [50] by different effects of the transfer channels on the complete fusion and by some problems with the experimental data analysis. 
Using the quantum diffusion approach, we try to reveal a systematic behavior of the complete fusion suppression as a function of the target charge $Z_{T}$ and colliding energy $E_{\text {c.m. }}$ and by comparing the calculated capture cross sections in the absence of breakup with the experimental complete fusion cross sections. Note that the effects of deformation and neutron transfer on the complete fusion suppression are taken into consideration as well. All calculated results are obtained with the same set of parameters as in Ref. [17]. The absolute values of the quadrupole deformation parameters $\beta_{2}$ of even-even deformed nuclei are taken from Ref. [25]. For the quadruple deformation parameter of an odd nucleus, we choose the maximal value of the deformation parameters of neighboring even-even nuclei. For the double magic and neighboring nuclei, in the ground state we set $\beta_{2}=0$.

The difference between the capture cross section and the complete fusion cross section can be ascribed to the breakup effect. Comparing $\sigma_{c}^{\text {th }}$ and $\sigma_{f u s}^{\text {exp }}$, one can estimate the breakup probability $P_{\mathrm{BU}}=1-\sigma_{\text {fus }}^{\text {exp }} / \sigma_{c}^{\text {th }}$. If at some energy $\sigma_{f u s}^{\text {exp }}>\sigma_{c}^{t h}$, the values of $\sigma_{c}^{t h}$ was normalized so to have $P_{\mathrm{BU}} \geq 0$ at any energy.

As seen in figures 7 and 8, at energies above the Coulomb barriers the values of $P_{\mathrm{BU}}$ vary from 10 to $60 \%$. In the reactions ${ }^{9} \mathrm{Be}+{ }^{144} \mathrm{Sm},{ }^{208} \mathrm{~Pb},{ }^{209} \mathrm{Bi}$ the value of $P_{\mathrm{BU}}$ increases with charge number of the target at $E_{\text {c.m. }}-V_{b}>3$ $\mathrm{MeV}$. This was also noted in Ref. [50]. However, the reactions ${ }^{9} \mathrm{Be}+{ }^{89} \mathrm{Y},{ }^{124} \mathrm{Sn}$ are out of this systematics. In the reactions ${ }^{6} \mathrm{Li}+{ }^{144} \mathrm{Sm},{ }^{198} \mathrm{Pt},{ }^{209} \mathrm{Bi}$ the value of $P_{\mathrm{BU}}$ decreases with increasing charge number of the target at $E_{\mathrm{c} . \mathrm{m} .}-V_{b}>$ $3 \mathrm{MeV}$. While in the reactions ${ }^{9} \mathrm{Be}+{ }^{89} \mathrm{Y},{ }^{144} \mathrm{Sm},{ }^{208} \mathrm{~Pb},{ }^{209} \mathrm{Bi}$ the value of $P_{\mathrm{BU}}$ has a minimum at $E_{\text {c.m. }}-V_{b} \approx 0$ and a maximum at $E_{\text {c.m. }}-V_{b} \approx-(1-3) \mathrm{MeV}$, in the ${ }^{9} \mathrm{Be}+{ }^{124} \mathrm{Sn}$ reaction the value of $P_{\mathrm{BU}}$ steady decreases with energy. In the reactions ${ }^{6} \mathrm{Li}+{ }^{144} \mathrm{Sm},{ }^{198} \mathrm{Pt},{ }^{209} \mathrm{Bi},{ }^{7} \mathrm{Li}+{ }^{208} \mathrm{~Pb},{ }^{209} \mathrm{Bi}$, and ${ }^{9} \mathrm{Li}+{ }^{208} \mathrm{~Pb}$ there is maximum of $P_{\mathrm{BU}}$ at $E_{\mathrm{c} . \mathrm{m} .}-V_{b} \approx-(0-1)$ $\mathrm{MeV}$. However, in the reactions ${ }^{6} \mathrm{Li}+{ }^{208} \mathrm{~Pb}$ and ${ }^{7} \mathrm{Li}+{ }^{165} \mathrm{Ho}$ $P_{\mathrm{BU}}$ has a minima $E_{\mathrm{c} . \mathrm{m} .}-V_{b} \approx 2 \mathrm{MeV}$ and no maxima at $E_{\text {c.m. }}-V_{b} \approx 0$. For ${ }^{9} \mathrm{Be}$, the breakup threshold is slightly larger than for ${ }^{6} \mathrm{Li}$. Therefore, we can not explain a larger breakup probability at smaller $E_{\mathrm{c} . \mathrm{m} .}-V_{b}$ in the case of ${ }^{9} \mathrm{Be}$. The possible explanation of it is that there are probably some problems with the data analysis which were earlier noted in Refs. $[48,50]$ from the point of view of a universal fusion function representation. It could be also that at energies near the Coulomb barrier the characteristic time of the breakup is larger than the characteristic time of the capture process and influences the complete fusion. For the reactions ${ }^{6,7} \mathrm{Li}+{ }^{208} \mathrm{~Pb}$, the characteristic times of the prompt and delayed breakup were studied recently in Ref. [51].

\section{Summary}

The quantum diffusion approach was applied to study the capture process in the reactions with deformed and spherical nuclei at sub-barrier energies. The available experimental data at energies above and below the Coulomb barrier are well described. As shown, the experimentally observed sub-barrier fusion enhancement is mainly related to the quadrupole deformation of the colliding nuclei and neutron transfer with large positive $Q$-value. The change of the magnitude of the capture cross section after the neutron transfer occurs due to the change of the deformations of nuclei. When after the neutron transfer the deformations of nuclei do not change or slightly decrease, the neutron transfer weakly influences or even suppresses the capture process. It would be interesting to study such-type of reactions.

It turns out that the sub-barrier capture (fusion) reactions with $Q_{1 n}<0$ and $Q_{2 n}>0$ may help in the understanding of the neutron pair tunneling phenomena and of the pair correlation in the surface of nucleus phenomena. The present work shows that the main contribution to transfer is due to the dineutron-like cluster component. In the capture process, the transfer of a neutron pair before the crossing of the Coulomb barrier is a clear signature of the strong correlations between the transferred nucleons affecting the dynamics and the surface character of pairing interaction. Our results indicate the dominance of the dineutron structure (of the preformed dineutron-like clusters) in the surface of the stable and unstable nuclei ${ }^{48} \mathrm{Ca}$, ${ }^{64} \mathrm{Ni}$, and ${ }^{116,124,132} \mathrm{Sn}$. Measurements of sub-barrier capture cross sections in various reactions can be utilized to study the role of pairing correlations between the transferred nucleons.

Comparing the calculated capture cross sections in the absence of breakup data and experimental complete fusion data, we analyzed the role of the breakup channels in the reactions with the light projectiles ${ }^{9} \mathrm{Be}$ and ${ }^{6,7,9} \mathrm{Li}$ at near-barrier energies. Within the quantum diffusion approach the neutron transfer and deformation effects were taken into account. Analyzing the extracted breakup probabilities, we showed that there are no systematic trends of breakup in the reactions studied. Moreover, for some system with larger (smaller) $Z_{T}$ we found the contribution of breakup to be smaller (larger).

Note that our conclusions coincide with those of Refs. $[48,50]$, where the universal fusion function formalism was applied for the analysis of experimental data. One needs to measure directly the breakup process in different systems, especially light ones, to understand the role of the Coulomb breakup in the complete fusion process. The other important subject to be investigated both experimental and theoretically is the characteristic time of the breakup. The first steps in these directions were done in Refs. [49,51, 52].

\section{References}

1. A.B. Balantekin, N. Takigawa, Rev. Mod. Phys. 70, 77 (1998)

2. L.F. Canto, P.R.S. Gomes, R. Donangelo, M.S. Hussein, Phys. Rep. 424, 1 (2006)

3. W. Henning, F.L.H. Wolfs, J.P. Schiffer, K.E. Rehm, Phys. Rev. Lett. 58, 318 (1987)

4. P.H. Stelson, H.J. Kim, M. Beckerman, D. Shapira, R.L. Robinson, Phys. Rev. C 41, 1584 (1990)

5. R. Pengo et al., Nucl. Phys. A411, 255 (1983)

6. R.B. Roberts et al., Phys. Rev. C 47, R1831 (1993)

7. A.M. Stefanini, D. Ackermann, L. Corradi, J.H. He, G. Montagnoli, S. Beghini, F. Scarlassara, G.F. Segato, Phys. Rev. C 52, R1727 (1995) 
8. R. Broglia, C.H. Dasso, S. Landowne, A. Winther, Phys. Rev. C 27, 2433 (1983); R. Broglia, C.H. Dasso, S. Landowne, G. Pollarolo, Phys. Lett. B 133, 34 (1983)

9. F. Scarlassara et al., EPJ Web of Conf. 17, 05002 (2011) 10. D.R. Bes, R.A. Broglia, Nucl. Phys. 80, 289 (1966); R.A. Broglia, O. Hansen, C. Riedel, Adv. Nucl. Phys. 6, 287 (1973)

11. R.V. Jolos, V.G. Kartavenko, F. Dönau, D. Janssen, Theor. Math. Fiz. 14, 70 (1973); R.V. Jolos and V.G. Kartavenko, Yad. Fiz. 19, 964 (1974); R.V. Jolos, V.G. Kartavenko, V. Rybarska, Theor. Math. Fiz. 20, 353 (1974); R.V. Jolos, V.G. Kartavenko, and S.M. Semenov, Yad. Fiz. 22, 1121 (1975)

12. W. von Oertzen, A. Vitturi, Rep. Prog. Phys. 64, 1247 (2001)

13. P.A. DeYoung at al., Phys. Rev. C 71, 051601 (2005)

14. T. Nakamura at al., Phys. Rev. Lett. 96, 252502 (2006)

15. P. Möller at al., Phys. Rev. Lett. 99, 252501 (2007)

16. A. Chatterjee at al., Phys. Rev. Lett. 101, 032701 (2008)

17. V.V. Sargsyan, G.G. Adamian, N.V. Antonenko, W. Scheid, Eur. Phys. J. A 45, 125 (2010)

18. V.V. Sargsyan, G.G. Adamian, N.V. Antonenko, W. Scheid, H.Q. Zhang, Eur. Phys. J. A 47, 38 (2011)

19. V.V. Sargsyan, Z. Kanokov, G.G. Adamian, N.V. Antonenko, W. Scheid, Phys. Rev. C 80, 034606 (2009); Phys. Rev. C 80, 047603 (2009)

20. G.G. Adamian, N.V. Antonenko, Z. Kanokov, V.V. Sargsyan, Teor. Mat. Fiz. 145, 87 (2005); Z. Kanokov, Yu.V. Palchikov, G.G. Adamian, N.V. Antonenko, W. Scheid, Phys. Rev. E 71, 016121 (2005); Yu.V. Palchikov, Z. Kanokov, G.G. Adamian, N.V. Antonenko, W. Scheid, Phys. Rev. E 71, 016122 (2005)

21. G.N. Knyazheva et al., Phys. Rev. C 75, 064602 (2007); A.M. Stefanini et al., Eur. Phys. J. A 23, 473 (2005)

22. J.R. Leigh et al., Phys. Rev. C 52, 3151 (1995)

23. M. Beckerman et al., Phys. Rev. C 28, 1963 (1983)

24. W. Reisdorf et al., Nucl. Phys. A438, 212 (1985)

25. S. Raman, C.W. Nestor Jr., P. Tikkanen, At. Data \& Nucl. Data Tables 78, 1 (2001)

26. J.M.B. Shorto et al., Phys. Rev. C 81, 044601 (2010)

27. D.E. DiGregorio et al., Phys. Rev. C 39, 516 (1989)

28. H. Timmers et al., Nucl. Phys. A633, 421 (1998)

29. A.J. Pacheco et al., Phys. Rev. C 45, 2861 (1992); R. Bock et al., Nucl. Phys. A388, 334 (1982)

30. G.G. Adamian, A.K. Nasirov, N.V. Antonenko, R.V. Jolos, Phys. Part. Nucl. 25, 583 (1994)

31. S. Szilner et al., Phys. Rev. C 76, 024604 (2007); L. Corradi, G. Pollarolo, and S. Szilner, J. Phys. G 36, 113101 (2009); Phys. Rev. C 84, 014325 (2011); L. Corradi et al., EPJ Web of Conf. 17, 08004 (2011)

32. H.Q. Zhang et al., Phys. Rev. C 82, 054609 (2010)

33. C.L. Jiang et al., Phys. Rev. C 82, 041601(R) (2010)

34. A.M. Stefanini et al., Eur. Phys. J. A 23, 1401 (1997); F. Scarlassara et al., Nucl. Phys. A672, 99 (2000)

35. E. Khan, N. Sandulescu, N.V. Giai, M. Grasso, Phys. Rev. C 69, 014314 (2004)

36. M. Matsuo and Y. Serizawa, Phys. Rev. C 82, 024318 (2010); H. Shimoyama and M. Matsuo, Phys. Rev. C 84, 044317 (2011)

37. E. Pllumbi, M. Grasso, D. Beaumel, E. Khan, J. Margueron and J. van de Wiele, Phys. Rev. C 83, 034613
(2011)

38. M. Grasso, D. Lacroix, A. Vitturi, Phys. Rev. C 85, 034317 (2012)

39. J.J. Kolata at al., Phys. Rev. C 75, 031302 (2007)

40. M. Assié and D. Lacroix, Phys. Rev. Lett. 102, 202501

(2009); J.A. Scarpaci at al., Phys. Rev. C 82, 031301(R) (2010)

41. M. Beckerman, M. Salomaa, A. Sperduto, J.D. Molitoris, A. DiRienzo, Phys. Rev. C 25, 837 (1982)

42. J.F. Liang et al., Phys. Rev. C 78, 047601 (2008)

43. D.J. Dean and M. Hjorth-Jensen, Rev. Mod. Phys. 75, 607 (2003)

44. C.A. Bertulani, EPJ Web Conf. 17, 15001 (2011)

45. N. Keeley, R. Raabe, N. Alamanos, J.L. Sida, Prog. Part. Nucl. Phys. 59, 579 (2007)

46. L.F. Canto, P.R.S. Gomes, J. Lubian, L.C. Chamon,

E. Crema, J. Phys. G 36, 015109 (2009)

47. L.F. Canto, P.R.S. Gomes, J. Lubian, L.C. Chamon, E. Crema, Nucl. Phys. A821, 51 (2009)

48. P.R.S. Gomes, J. Lubian, L.F. Canto, Phys. Rev. C 79, 027606 (2009)

49. R. Rafiei et al., Phys. Rev. C 81, 024601 (2010)

50. P.R.S. Gomes, R. Linares, J. Lubian, C.C. Lopes, E.N. Cardozo, B.H.F. Pereira, I. Padron, Phys. Rev. C 84, 014615 (2011)

51. D.H. Luong et al., Phys. Lett. B 695, 105 (2011); EPJ

Web Conf. 17, 03002 (2011)

52. P.R.S. Gomes, private communication (2012) 